\title{
Optimizing End-to-End Throughput for Data Transfers on an Overlay-TCP Path ${ }^{\star}$
}

\author{
Pradnya Karbhari, Mostafa Ammar, and Ellen Zegura \\ College of Computing, Georgia Institute of Technology, Atlanta, GA-30332 \\ \{pradnya, ammar, ewz\}@cc.gatech.edu
}

\begin{abstract}
We consider an overlay network where traffic on an overlay hop is carried in one or more TCP connections established between the overlay nodes at the ends of the hop. We are interested in maximizing the throughput of data carried by this type of overlay network. To that end, we focus on a single path in such a network and investigate how one can determine the number of TCP connections on each overlay hop so as to maximize the throughput of the data carried end-to-end on that path. We first show that having more than one TCP connection on some overlay hops can indeed increase the throughput on overlay paths. We then propose the Adaptive Overlay-TCP Provisioning approach, that, based on the path state, dynamically introduces and removes TCP connections on individual overlay hops to maximize throughput while minimizing the overhead of extraneous connections. We consider two schemes to assess the path state, the intermediate buffer occupancy scheme and the isolated rate probing scheme, and evaluate them experimentally on a set of Planetlab nodes. We show that these schemes can significantly improve the end-to-end throughput with very little overhead.
\end{abstract}

Keywords: Overlay networks, parallel TCP connections.

\section{Introduction}

Overlay networks have been proposed for a variety of applications such as content distribution [1], multicast [2, quality of service [3] and multimedia streaming [4]. Ideally, these multiple overlay networks should coexist without harming each other. In particular, it is important that coexisting overlay networks share native network link bandwidths in a fair manner, a problem identified in [5]. One way to achieve this is to carry data on overlay hops using one or more TCP connections between overlay nodes at the ends of the hops. We refer to such overlay networks as Overlay-TCP network $\$$. Such networks have the desirable property that they ensure that multiple overlays will share native link bandwidths fairly, and that

\footnotetext{
* This work is supported by NSF grant ANI-0240485.

${ }^{1}$ In this work, we consider overlays that provide a permanent and tunable infrastructure to support applications. We do not look at overlays resulting from peer-to-peer systems that have higher churn rate amongst nodes and hence amongst overlay links.
} 


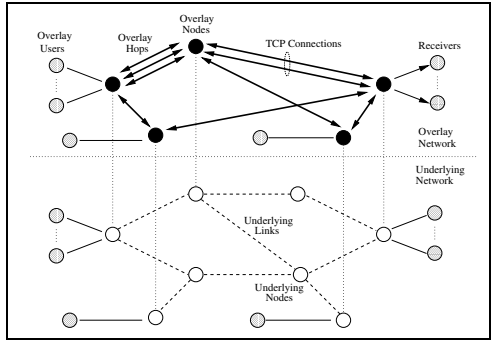

Fig. 1. Overlay Network

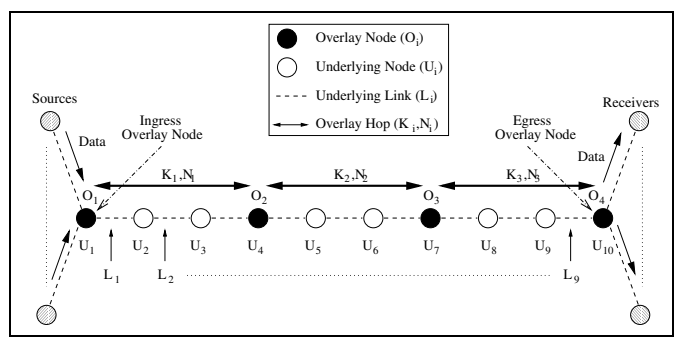

Fig. 2. Model for Overlay Path

the Internet will not suffer due to lack of congestion control in the network. Figure 1 shows an example of an Overlay-TCP network.

Our ultimate goal is to maximize the throughput of data carried on OverlayTCP networks. To that end, in this paper, we focus on the problem of maximizing the throughput on a path in such an overlay network. We assume the existence of a separate routing infrastructure that determines the overlay path from a sender $S$ to a receiver $R$. The main design parameter in this work is the number of TCP connections on each overlay hop in the path. To understand the need for such a design, we define the isolated rate of an overlay hop as the throughput that a single long-lived continuously backlogged TCP connection would achieve on that hop. Now consider an Overlay-TCP path in which a single TCP connection is used on each hop. Clearly, the isolated rates on the overlay hops are usually not equal, and vary over time. The end-to-end throughput on this path is limited by the minimum of the isolated rates on the individual overlay hops.

This end-to-end throughput can be improved by introducing multiple parallel connections on slower overlay hops to increase their rate to match that of faster overlay hops. This might be considered an aggressive measure, and unfair to connections competing on the bottleneck native link. However, the addition of a bound on the number of parallel connections can provide effective control on this unfairness and equalize treatment among multiple overlays. Also, we are using TCP connections, which are congestion responsive. Finally, although we intend to use parallel connections on overlay hops, we aim to maximize the throughput using as few connections as possible.

The improvement in end-to-end throughput achieved with the use of multiple parallel connections comes at the expense of packet reordering at the receiver. We observed experimentally that the reordering index 2 increases with increasing number of parallel connections between two nodes, especially for overlay paths of more than 2 hops. The receiver has to buffer and reorder all data sent over multiple parallel connections, thus adding some complexity. Thus, packet reordering is an added incentive to keep the number of parallel connections to a minimum.

${ }^{2}$ We define the packet reordering index as the root mean square error of the received packet order compared to the initial sending order. 
We first show that using more than one connection on some overlay hops does indeed increase the throughput on overlay paths. We then propose the Adaptive Overlay-TCP Provisioning approach using two schemes (intermediate buffer occupancy scheme and isolated rate probing scheme) that assess the path state and dynamically introduce and remove TCP connections on individual overlay hops. We evaluate these schemes on a set of PlanetLab [6] nodes 3 and show that our schemes significantly increase the end-to-end throughput with as few extraneous connections as possible. We discuss the appropriate parameters to be used so as to be less aggressive while maximizing the end-to-end throughput.

Analysis of TCP connections on consecutive overlay hops has been undertaken in various contexts in quite a few papers [8, 9, 10,11. All these papers employ schemes such as TCP backpressure, packet dropping, and explicit congestion notification to throttle connections to the rate of the slowest hop. None of these consider the option of using multiple TCP connections on overlay hops. Amir et al. 11] mention the possibility of multiple connections on each hop, but do not explore it further. In the context of reliable multicast, a number of studies [2,12,13 show that the end-to-end throughput of an overlay path, and consequently the whole multicast tree is limited by the overlay hop with the minimum throughput. The effect of using end-to-end parallel TCP connections for transferring data on a single path, or from multiple servers on multiple paths has been studied earlier 14,15,16, but not on overlay paths of two or more hops.

The outline of the paper is as follows. We formulate the problem in Section 2 and present a case study of a 2-hop overlay path in Section 3. We discuss the proposed Adaptive Overlay-TCP Provisioning architecture in Section 4, outline the proposed schemes in Section [5, and evaluate their performance in Section 6] In Section [7, we summarize the conclusions and discuss future work.

\section{Problem Statement}

Our model for an overlay path is shown in Figure2, The parameters and variables for the overlay path are listed in Table 2 . Consider a path of $m$ nodes, each node denoted by $U_{i}$ in the underlying network. These nodes are connected by $m-1$ links, each denoted by $L_{i}$. Let $n$ of the $m$ underlying nodes function as overlay nodes, where $n \leq m$. The overlay nodes, denoted by $O_{i}$, form an overlay path of $n-1$ hops, each denoted by $K_{i}$, with $N_{i}$ TCP connections on each hop. The overlay path comprises one or more links and nodes in the underlying network.

We consider one-way data transfer on this path. A set of sources send data to a set of receivers. The senders and receivers need not be part of the overlay network. Sources send data to an ingress overlay node, which detects the transfer, say by looking at headers, and then forwards it over the Overlay-TCP network, as

${ }^{3}$ We realize that PlanetLab, like any other experimental infrastructure, is not completely representative of the real Internet 7]. However, it is currently the best infrastructure available to researchers to evaluate their work in a relatively realistic scenario on the Internet. 
Table 1. Model of an Overlay Path

\begin{tabular}{|l|l|}
\hline \multicolumn{2}{|l|}{ System Parameters } \\
\hline \hline$m$ & Number of underlying nodes on the path \\
\hline System & Variables \\
\hline \hline$U_{i}$ & $i^{t h}$ underlying node on the path, $i=1 . . m$ \\
$O_{i}$ & $i^{t h}$ overlay node on the path, $i=1 . . n$ \\
$L_{i}$ & $i^{t h}$ underlying link on the path, $i=1 . . m-1$ \\
$K_{i}$ & $i^{\text {th }}$ overlay hop on the path, $i=1 . . n-1$ \\
$N_{i}$ & Number of TCP connections on $i^{t h}$ overlay hop on the path, $i=1 . . n-1$ \\
$R_{i}$ & Average isolated rate on $i^{t h}$ overlay hop on the path, $i=1 . . n-1$ \\
\hline
\end{tabular}

suggested by the proxy mechanism in 17 . The egress overlay node demultiplexes the data and delivers it to the appropriate receiver. We assume that the aggregate incoming data at the ingress overlay node keeps the connection continuously backlogged. The overlay path is assumed to carry background traffic.

Given such an overlay path, with varying background traffic, our goal in this work is to dynamically determine and provision the number of TCP connections necessary on each overlay hop in order to maximize the end-to-end throughput. We refer to this approach as Adaptive Overlay-TCP Provisioning.

\section{Case Study: 2-Hop Overlay Path}

We start by studying the behavior of a 2-hop overlay path with a single connection on each hop. At the intermediate overlay node, the socket buffer for the incoming TCP connection forwards the data packets to an application layer buffer. This buffer then forwards the packets to the socket buffer for the outgoing TCP connection. See Figure 4 for more details.

Recall that we define the isolated rate of an overlay hop as the throughput that a single long-lived continuously backlogged TCP connection would achieve on that overlay hop. The isolated rates of the incoming hop $\left(R_{1}\right)$ and the outgoing hop $\left(R_{2}\right)$ at any overlay node will generally be unequal. We define the degree of mismatch, $M(M \geq 1)$ at an overlay node as $M=\frac{\max \left(R_{1}, R_{2}\right)}{\min \left(R_{1}, R_{2}\right)}$.

1) If $R_{1}<R_{2}$, data comes in at the intermediate node at a rate slower than what can be forwarded on the outgoing hop. The outgoing connection does not have enough data to operate at its isolated rate $\left(R_{2}\right)$ and is limited by the data rate of the incoming connection. Also, since it is not continuously backlogged any more, it might repeatedly go into slow start and get an even lower throughput.

2) If $R_{1}>R_{2}$, data comes in at the intermediate node at a rate faster than what the outgoing connection can keep up with. Although the outgoing connection can now operate at the maximum rate $\left(R_{2}\right)$, the intermediate node has to 


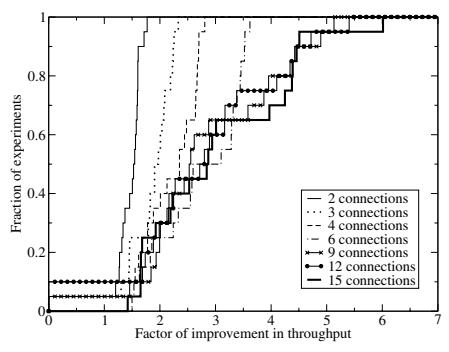

Fig. 3. Improvement in throughput using multiple connections

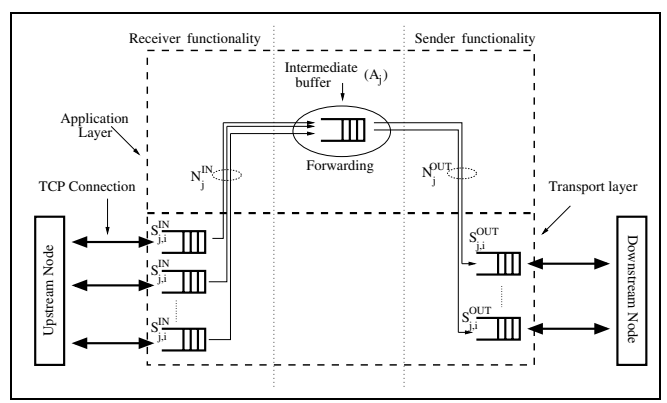

Fig. 4. Model for Intermediate Overlay Node $O_{j}$

buffer the excess data. Once the buffers at the intermediate node are full, the incoming connection has to throttle back. It can send more data only after the buffers have space to hold it. The incoming connection is now bounded by the data rate of the outgoing connection. Also, now the incoming connection might go into repeated slow-starts, thus lowering the throughput even more.

3) If $R_{1}=R_{2}$, the average connection rates over some time period $T$ are equal. However, at any instant, each TCP connection will be in the slow start or congestion avoidance phase. These phases need to be synchronized in order to get the maximum effective throughput.

Effectively, the end-to-end throughput on a 2-hop overlay path with a single TCP connection on each hop is bounded by the throughput of the slower connection on the path. In an attempt to improve the end-to-end throughput, we evaluate the effect of using multiple TCP connections on the slower overlay hop.

We set up 2-hop overlay paths on a set of Planetlab [6] nodes and determined the isolated rates on each hop by performing a $50 \mathrm{MB}$ transfer on a TCP connection between consecutive nodes. We then started data transfers with one TCP connection on the faster hop and multiple (2-15) connections on the slower hop. Figure 3 shows a sample of results obtained on an overlay path where nodes at Duke, Georgia Tech (GT) and U-Arizona were used as ingress, intermediate and egress overlay nodes respectively. On this path, the isolated rate from Duke to GT was higher than that between GT and U-Arizona $\left(R_{1}>R_{2}\right)$. The figure plots a CDF of the factor of improvement, defined as the ratio of the end-to-end throughput using multiple connections on an overlay hop, to that using a single connection on each hop. We see that the factor of improvement increases with the number of downstream connections. However, the effect of adding new connections decreases as the number of connections increase. We observed similar results for the $R_{1}<R_{2}$ case, with multiple connections on the incoming hop.

We conclude from these experiments that adding multiple TCP connections on a slower hop does improve end-to-end throughput. However, beyond a threshold, adding new connections does not have a significant effect on the throughput. 
Table 2. Adaptive Overlay-TCP Provisioning: Parameters and Variables

\begin{tabular}{|l|l|}
\hline \hline Parameters \\
\hline \hline$N_{\max }$ & Maximum number of TCP connections between consecutive overlay nodes \\
$A_{j}$ & Size of application layer buffer \\
$S_{j, i}^{I N}$ & Size of socket buffer for each incoming connection \\
$S_{j, i}^{O U T}$ & Size of socket buffer for each outgoing connection \\
\hline \hline \multicolumn{2}{|l|}{ Variables at Overlay Node $O_{j}$} \\
\hline \hline$M_{j}$ & Degree of mismatch between upstream and downstream isolated rates \\
$N_{j}^{I N}$ & Current number of incoming TCP connections \\
$N_{j}^{O U T}$ & Current number of outgoing TCP connections \\
$B_{j, i}^{I N}$ & Current occupancy of socket buffer for each incoming connection \\
$B_{j, i}^{O U T}$ & Current occupancy of socket buffer for each outgoing connection \\
\hline
\end{tabular}

\section{The Adaptive Overlay-TCP Provisioning Architecture}

Generalizing the above discussion to an $n$-hop overlay path, the effective end-toend throughput of data transfers on this path, with a single TCP connection on each hop, will be limited by the slowest TCP connection on the path. We can improve the throughput by using multiple TCP connections on slower hops.

The architecture for a system to provide Adaptive Overlay-TCP Provisioning is based on the model of an overlay node $O_{j}$, shown in Figure 4. An overlay node, denoted by the dashed box, is split into the Application and Transport layers. A pair of consecutive overlay nodes can communicate over a maximum of $N_{\max }$ TCP connections. $N_{\max }$ is a system-wide parameter. At any point of time, the node $O_{j}$ has $N_{j}^{I N}$ active TCP connections with the upstream node, and $N_{j}^{O U T}$ active TCP connections with the downstream node. These connections read or write data into transport layer socket buffers, each with a maximum capacity of $S_{j, i}^{I N}$ and $S_{j, i}^{O U T}$ for incoming and outgoing TCP connections respectively. All incoming connections forward packets from the socket buffers to the application buffer, of capacity $A_{j}$. Effectively, each node has a buffering capacity of $\sum_{i=1}^{N_{\max }} S_{j, i}^{I N}+\sum_{i=1}^{N_{\max }} S_{j, i}^{O U T}+A_{j}$. For each node $j, B_{j, i}^{I N}$ and $B_{j, i}^{O U T}$ denote the instantaneous incoming and outgoing socket buffer occupancy for connection $i$.

The proposed architecture consists of the following three components:

1) Network Condition Evaluation Module: This module evaluates network conditions under which the TCP connections operate by measuring one of two quantities. A direct approach is to periodically probe the isolated rates on each hop by performing a data transfer. We can also indirectly measure the relative isolated rates by measuring the buffer occupancy of incoming TCP connections.

2) The Decision Algorithm: Based on the measured quantities, the decision algorithm decides whether multiple connections are required on any hop on the overlay path. If yes, it also gives either the number of connections 


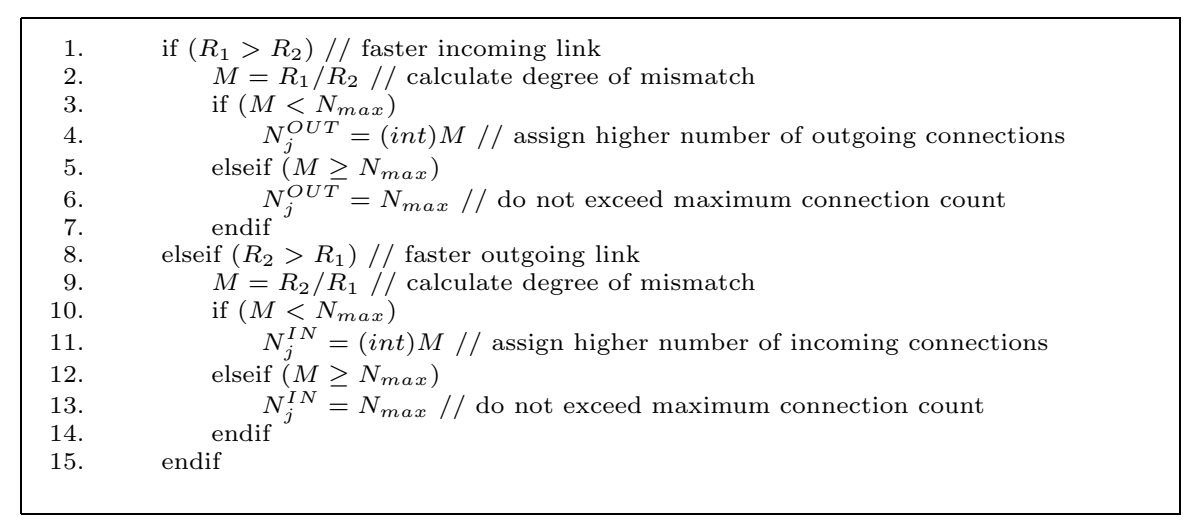

Fig. 5. Decision Algorithm using Isolated Rates

required on each hop, or a decision to increase or decrease the number of connections.

3) Connection Setup and Maintenance: The varying number of upstream and downstream connections can be implemented in two ways. First, we can keep $N_{\max }$ connections active at all times. At any point of time, we use $N_{j}^{I N}$ and $N_{j}^{O U T}$ connections, as computed by the decision algorithm, to send data. Another option is to start a new connection and tear down an existing one every time the decision algorithm alters either $N_{j}^{I N}$ or $N_{j}^{O U T}$. Due to the connection setup overhead associated with this option, we use the first option in our solution.

The number of connections required on each hop might change over the course of the data transfer due to changing network conditions. Hence, in our schemes, we periodically evaluate the network conditions and repeat the decision process.

\section{$5 \quad$ Proposed Schemes}

Based on the two quantities measured by the network condition evaluation module, we propose two schemes and corresponding decision algorithms to determine the number of connections required on each overlay hop.

\subsection{Direct Measurement Approach: Isolated Rate Probing Scheme}

In this scheme, we directly measure the isolated rate on each overlay hop by periodically performing a $250 \mathrm{~KB}$ transfer on a TCP connection on that hop. Although this scheme gives a relatively accurate estimate of the isolated rates, it places additional load on the path of the data transfer. We can use the lightweight alternative of measuring loss probability and round-trip time for each hop, and estimating the TCP throughput using the TCP equation [18] to give an estimate of the isolated rates. In this work we do not implement this alternative. 


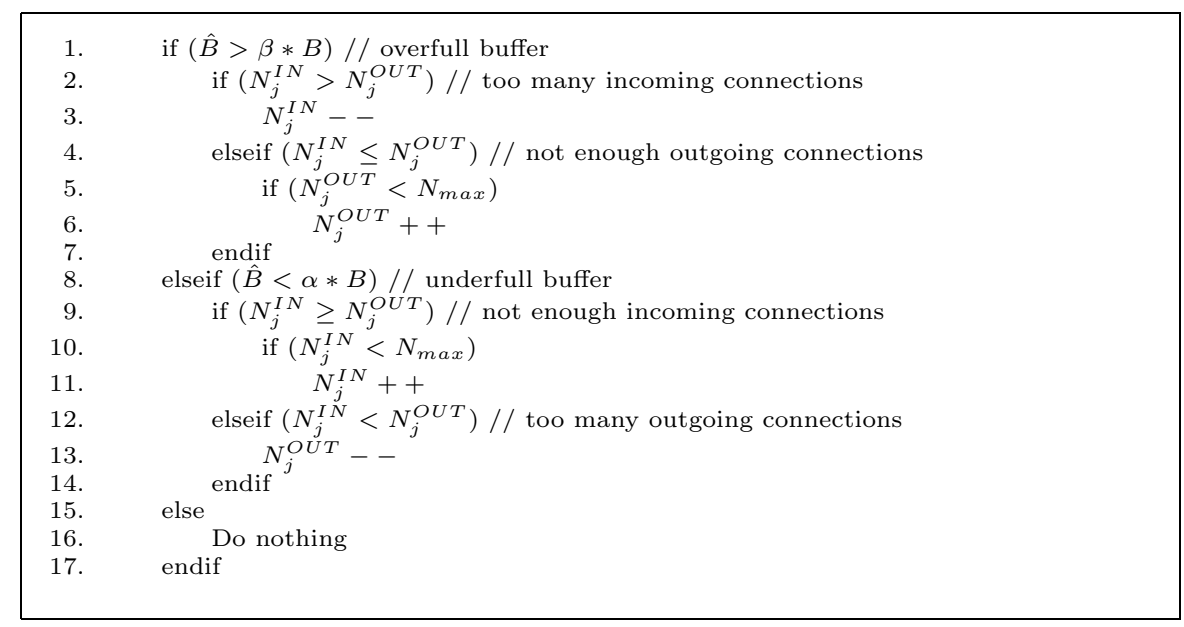

Fig. 6. Decision Algorithm using Buffer Estimates

2-Hop Overlay Path: Figure 5 shows the decision algorithm at overlay node $O_{j}$ to determine the location and number of connections on a 2-hop overlay path, with isolated rates $R_{1}$ and $R_{2}$ on incoming and outgoing hops respectively. If $R_{1}$ is greater, the algorithm computes the degree of mismatch $(M)$ in Line 2 , which is the required number of outgoing connections (Line 4). Lines 8-14 handle the case when $R_{2}$ is greater. Lines 5 and 12 ensure that $N_{\max }$ is not exceeded.

Multi-hop Overlay Path: We now extend the algorithm to the multihop case. We compute $M_{i}^{\max }$, the maximum degree of mismatch for each hop along the overlay path as the ratio of the maximum isolated rate $\left(R_{\max }\right)$ on the path, to the isolated rate $\left(R_{i}\right)$ of that hop. This ratio gives the number of connections required on that hop. Taking $N_{\max }$ into account, the number of downstream connections required at each node is given by $N_{i}^{O U T}=\min \left(\frac{R_{\max }}{R_{i}}, N_{\max }\right)$. To implement this algorithm, the ingress node sends its downstream isolated rate to its downstream node, which appends its own downstream isolated rate and forwards to the next node and so on. Once the vector reaches the egress node, it computes $N_{i}^{O U T}$ for each hop and sends the vector back along the path.

\subsection{Intermediate Buffer Occupancy Scheme}

The above scheme can incur a significant overhead for determining isolated rates and signaling among overlay nodes. We now describe a scheme based on local observation of an overlay node's buffers. To illustrate this scheme, consider a 2-hop overlay path where the isolated rate on the incoming hop is greater than that on the outgoing hop $\left(R_{1}>R_{2}\right)$. In this case, the buffer will be relatively full most of the time, whereas, if $R_{1}<R_{2}$, the buffer will be relatively empty. We validated this by observing the incoming socket buffer occupancy over time for several 2-hop overlay paths on a set of PlanetLab nodes.

For the general multi-hop case, Figure 6 shows the decision algorithm at an overlay node to determine whether it needs to either increase or decrease the 
number of connections on the incoming or outgoing hop. This algorithm operates at each overlay node independently. It uses a buffer occupancy estimator, $\hat{B}$, based on an exponentially weighted moving average of periodic samples of instantaneous buffer occupancy as follows, $\hat{B}=\gamma * \hat{B}+(1-\gamma) * \hat{B}_{\text {sample, }}$, where $0<\gamma<1$. Let $\alpha$ and $\beta$ be the low and high watermarks respectively, below which we consider the buffer to be underfull, and above which we consider it to be overfull. These parameters are the fraction of the total buffer space available at the receiving end of the incoming TCP connection at the intermediate node.

If the buffer is overfull, the algorithm checks whether the number of incoming $\left(N_{j}^{I N}\right)$ or outgoing connections $\left(N_{j}^{O U T}\right)$ is higher. If $N_{j}^{I N}$ is too high (Line 2), the outgoing link might be unable to keep up, hence we decrease $N_{j}^{I N}$. If $N_{j}^{I N}$ is less than $N_{j}^{\text {OUT }}$ (Line 4), the outgoing connections cannot keep up even with very few incoming incoming connections, hence we increase $N_{j}^{O U T}$. Line 5 ensures that this number does not exceed $N_{\max }$ at any point of time. Lines 8-14 handle the case when the buffer is underfull. If the current buffer occupancy is within the acceptable range $(\alpha * B \leq \hat{B} \leq \beta * B)$, we do not take any action.

At any overlay node, a change in $N_{j}^{O U T}$ is implemented immediately. However, a change in $N_{j}^{I N}$ is communicated to the upstream node, which changes its $N_{j}^{\text {OUT }}$ if its own decision algorithm concurs. The ingress node implements any decision by its downstream node, as it does not do any buffer estimation itself.

With this scheme, although we can measure network conditions without introducing additional traffic and do the estimation while the data transfer is in progress, we cannot determine exact values of the isolated rates, and hence we do not know the exact number of connections required. We need to continuously monitor and increase or decrease the number of connections by one every time.

\section{Performance Evaluation}

In this section, we present a set of experimental results, based on experiments on Planetlab [6] nodes. We discuss the measurement methodology, the parameters for the schemes, and then the performance results.

\subsection{Measurement Methodology}

We experimented with multiple overlay paths of 2-5 hops, by selecting 3-6 overlay nodes for each path from the set of available Planetlab [6] nodes. For the isolated rate probing scheme, we first started a set of control processes that performed a $250 \mathrm{~KB}$ transfer every 30 seconds to estimate the current value of $R_{i}$. We then started parallel transfers of $100 \mathrm{MB}$ each between the following nodes.

1) Direct TCP transfer between all sets of consecutive overlay nodes. These are used to monitor the isolated rates on each overlay hop.

2) Overlay-TCP transfer from ingress overlay node to egress overlay node with a single TCP connection on each overlay hop. We compare the throughput of data transfers using our schemes to this benchmark case, which would be the one used in an Overlay-TCP path without multiple connections. 


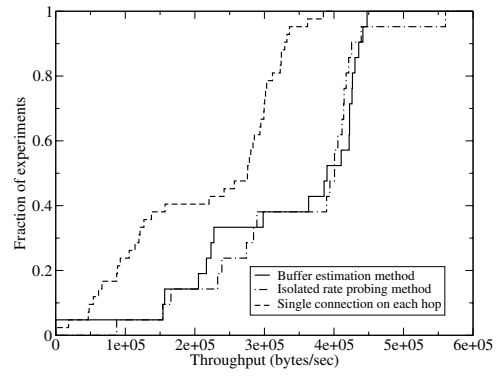

(a) End-to-End Throughput

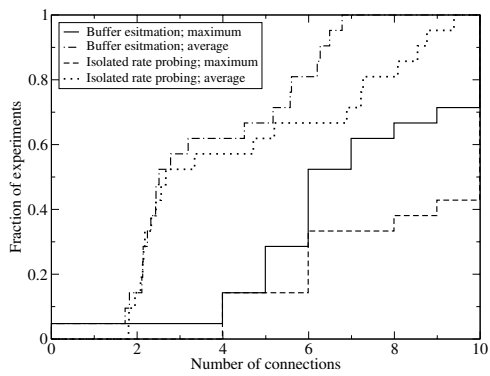

(b) Number of Connections

Fig. 7. 2-hop overlay path

3) Overlay-TCP transfer from ingress overlay node to egress overlay node using our Adaptive Overlay-TCP Provisioning schemes to maintain the appropriate number of parallel connections between overlay nodes.

\subsection{Parameters}

The parameters involved in the implementation and the choice of appropriate values for each parameter, based on our experiments, are discussed below.

Socket Buffer Size: Our experiments with the TCP socket buffer size varying from minimum $(8 \mathrm{~KB})$ to maximum $(256 \mathrm{~KB})$ showed that the end-to-end throughput of a data transfer over an Overlay-TCP path with a single connection on each hop is higher with the socket buffer size and the receiver advertised window set to the maximum value. This is because the socket buffers can absorb intermittent bursts in either the upstream or downstream connections.

$E W M A$ parameter $(\gamma): \gamma$ is the fractional weight of the previous buffer estimate in the exponentially weighted moving average estimator for estimating the current buffer occupancy of the incoming TCP connections. We experimented with values of $\gamma$ from 0.05 to 0.95 , and concluded that a value between the range 0.7 to 0.95 follows the trend well enough and filters out intermediate bursts in the buffer occupancy. In our experiments we used $\gamma=0.85$.

Estimation and Decision Algorithm Time Interval: In our experiments, we estimate the buffer occupancy every $200 \mathrm{~ms}$. We run the decision algorithm every 30 seconds to determine whether the network conditions have changed enough to either increase or decrease the number of outgoing or incoming connections.

Buffer Occupancy Thresholds ( $\alpha$ and $\beta$ ): The buffer occupancy thresholds determine how aggressive the scheme is in using parallel connections. The lower threshold is set to 0.1 , below which we consider the socket buffers to be underfull. In order to set the higher threshold, we observed from our experiments on 2-hop paths that if $\beta$ is too low (0.15), a large number of connections are added and removed on the downstream hop, even when $R_{1}<R_{2}$. On the other hand, 


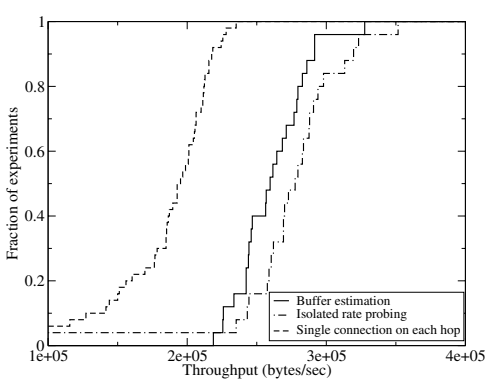

(a) End-to-End Throughput

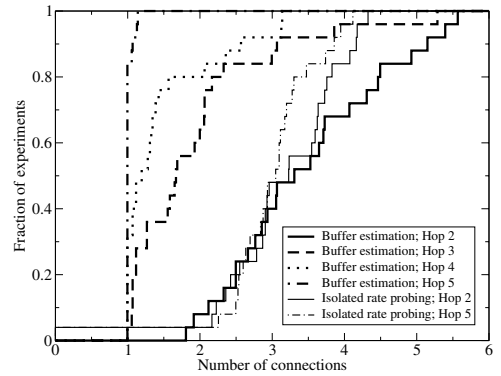

(b) Number of Connections

Fig. 8. 5-hop overlay path

if $\beta$ is too high (0.8), the addition of connections is very slow, even when the downstream overlay hop is the bottleneck. In our experiments, we use $\beta=0.4$.

Maximum connections on each overlay hop $\left(N_{\max }\right)$ : We use $N_{\max }=10$ in our experiments, based on results shown in Section 3 .

\subsection{Evaluation for a 2-Hop Overlay Path}

We performed experiments, as described in Section 6.1 using parameters listed above on a variety of 2-hop overlay paths. On each path, we performed 10-25 experiments. We present representative results for an overlay path with Cornell Univ, Columbia Univ and Stanford Univ as ingress, intermediate and egress nodes respectively. Figure 7(a) shows a CDF of the end-to-end throughput achieved using the proposed schemes and that achieved using a single connection on each hop. The two schemes show similar performance, with the isolated rate probing scheme achieving a slightly higher throughput. Figure 7(b) shows a CDF of average and maximum number of connections started by each scheme. The isolated rate probing scheme is more aggressive, and uses more maximum as well as average connections. The buffer estimation scheme is more conservative and thus achieves lower throughput. We also observed that an increase in degree of mismatch causes a higher factor of improvement in throughput, using either of the two schemes, compared to using a single connection on each hop.

\subsection{Evaluation for a Multi-hop Overlay Path}

We evaluated the two schemes on multiple overlay paths of 3-5 hops. Figure 8(a) shows a CDF of the throughput achieved using the two schemes, and that achieved using a single TCP connection on each hop, for a sample 5 hop overlay path between Univ of Massachusetts, New York Univ, Georgia Tech, U-Texas, UArizona and UCLA. Clearly, the two proposed schemes achieve better throughput than that using a single TCP connection on each hop. The isolated rate probing scheme achieves higher throughput than the buffer estimation scheme. 
Figure $8(\mathrm{~b})$ shows a CDF of the average number of connections started by each scheme on different overlay hops. The isolated rate probing scheme starts 2 connections on hops $1,3,4$, and the buffer estimation scheme starts 1 connection on hop 1. On the bottleneck hop (hop 2), the buffer estimation scheme starts more connections than the isolated rate probing scheme. On other hops, the isolated rate probing scheme is again more aggressive than the buffer estimation scheme.

\section{Conclusion}

The end-to-end throughput of data transfers in overlay networks that carry data over one or more TCP connections between consecutive overlay nodes is limited by the minimum of the TCP throughputs achievable on each overlay hop. In this work, we aim to maximize the end-to-end throughput of a data transfer on a path in an Overlay-TCP network by using multiple parallel connections on one or more overlay hops. We show that the use of multiple parallel connections on some hops does indeed increase the end-to-end throughput. We propose two schemes that assess the network conditions and dynamically adjust the number of connections used on each overlay hop. We show through experiments on Planetlab nodes that both schemes significantly improve performance while keeping the number of extraneous connections to a minimum. The overlay path design was a first step toward our plan of designing an Overlay-TCP network with the aim of maximizing the throughput of data carried by the entire overlay network. We also plan to study the use of multiple connections on multiple paths between two overlay nodes and its effect on end-to-end throughput. We further aim to study the fair sharing of native link bandwidth between multiple overlay networks.

\section{References}

1. Apostolopoulos, J., Wong, T., Wee, S., Tan, D.: On Multiple Description Streaming with Content Delivery Networks. In: IEEE Infocom. (2002)

2. Kwon, G., Byers, J.: Roma: Reliable overlay multicast with loosely coupled tcp connections. In: IEEE Infocom. (2004)

3. Subramanian, L., Stoica, I., Balakrishnan, H., Katz, R.: OverQos: An Overlay based Architecture for Enhancing Internet QoS. In: NSDI. (2004)

4. Padmanabhan, V., Wang, H., Chou, P., Sripanidkulchai, K.: Distributing Streaming Media Content Using Cooperative Networking. In: NOSSDAV. (2002)

5. Keralapura, R., Taft, N., Chuah, C., Iannaccone, G.: Can ISPs take the heat from Overlay Networks? In: HotNets-III. (2004)

6. PlanetLab. (http://www.planet-lab.org/)

7. Banerjee, S., Griffin, T., Pias, M.: The Interdomain Connectivity of Planet lab Nodes. In: Passive and Active Measurement Workshop. (2004)

8. Sundararaj, A., Duchamp, D.: Analytical Characterization of the Throughput of a Split TCP Connection. In: Stevens Inst. Tech Report. (2003)

9. Swany, M., Wolski, R.: Improving throughput with cascaded tcp connections: the logistical session layer. In: UCSB Tech Report. (2002) 
10. Lee, B., Balan, R., Jacob, L., Seah, W., Ananda, A.: Avoiding Congestion Collapse on the Internet using TCP Tunnels. Computer Networks (2002)

11. Amir, Y., Danilov, C.: Reliable communication in overlay networks. In: International Conference on Dependable Systems and Networks. (2003)

12. Baccelli, F., Chaintreau, A., Liu, Z., Riabov, A., Sahu, S.: Scalability of reliable group communication using overlays. In: IEEE Infocom. (2004)

13. Urvoy-Keller, G., Biersack, E.: A congestion control model for multicast overlay networks and its performance. In: Networked Group Communication. (2002)

14. Balakrishnan, H., Rahul, H., Seshan, S.: An Integrated Congestion Management Architecture for Internet Hosts. In: ACM SIGCOMM. (1999)

15. Rodriguez, P., Kirpal, A., Biersack, E.: Parallel-Access for Mirror Sites in the Internet. In: IEEE Infocom. (2000)

16. Gkantsidis, C., Ammar, M., Zegura, E.: On the effect of large-scale deployment of parallel downloading. In: IEEE Workshop on Internet Applications. (2003)

17. Peterson, L., Shenker, S., Turner, J.: Overcoming the Internet Impasse through Virtualization. In: HotNets-III. (2004)

18. Padhye, J., Firoiu, V., Towsley, D., Kurose, J.: Modeling TCP Throughput: A Simple Model and its Empirical Validation. In: ACM SIGCOMM. (1998) 